AperTO - Archivio Istituzionale Open Access dell'Università di Torino

\title{
Ab initio Hartree-Fock study of tetragonal and cubic phases of zirconium dioxide
}

\section{This is the author's manuscript}

Original Citation:

Availability:

This version is available http://hdl.handle.net/2318/102707

since

Published version:

DOI:10.1103/PhysRevB.45.592

Terms of use:

Open Access

Anyone can freely access the full text of works made available as "Open Access". Works made available under a Creative Commons license can be used according to the terms and conditions of said license. Use of all other works requires consent of the right holder (author or publisher) if not exempted from copyright protection by the applicable law. 


\title{
$A b$ initio Hartree-Fock study of tetragonal and cubic phases of zirconium dioxide
}

\author{
R. Orlando, C. Pisani, and C. Roetti \\ Department of Inorganic, Physical, and Materials Chemistry, University of Torino, via Giuria 5, \\ I-10125 Torino, Italy \\ E. Stefanovich \\ Department of Condensed Matter Chemical Physics, Latvia University, Rainis Boulevard, 19, \\ Riga 226098, U.S.S.R. \\ (Received 17 April 1991)
}

\begin{abstract}
The structural and electronic properties of cubic and tetragonal zirconia $\left(\mathrm{ZrO}_{2}\right)$ are studied using a periodic $a b$ initio Hartree-Fock method. Only valence electrons are treated explicitly, while effective core potentials are used for describing core electrons. The equilibrium geometries and elastic properties of the two phases are studied, and the mechanism of phase transition is discussed, with possible effects of applied pressure being taken into consideration. The analysis of the electronic structure shows appreciable departure from a purely ionic type of bond.
\end{abstract}

\section{INTRODUCTION}

Zirconia is an important material in ceramic engineering and recently its significance was proved in catalytic studies. ${ }^{1}$ Although the physical properties of zirconia are a subject of intensive theoretical and experimental study (the reader is referred to a recent book ${ }^{2}$ and to a collection of papers in a special journal issue devoted to ceramic materials ${ }^{3}$ ), many of its features are not as yet clearly understood. One of them is the stability of different crystalline phases of this compound at different temperatures and pressures. This knowledge of phase stability and transformations is of great importance for improving the characteristics of ceramic materials. ${ }^{4}$

It is established that $\mathrm{ZrO}_{2}$ has three zero-pressure modifications. At low temperatures the monoclinic $C_{2 h}^{5}$ $\left(P 2_{1} / c\right)$ phase (baddeleyite) is stable. ${ }^{5}$ Around $1400 \mathrm{~K}$ there is a phase transition to tetragonal $D_{4 h}^{15}\left(P 4_{2} / n m c\right)$ structure. ${ }^{6}$ At a temperature of about $2600 \mathrm{~K}$, the tetragonal phase transforms into the cubic fluorite $O_{h}^{5}(\mathrm{Fm} 3 \mathrm{~m})$ structure: ${ }^{7}$ This cubic structure is a special case of the tetragonal structure and can be obtained from the latter by making the ratio of the lattice constants $c / a$ equal to $2^{1 / 2}$ and by shifting pairs of oxygen atoms in the $z$ direction to their central positions in the unit cell (see Fig. 1). Various modifications have been found at high pressures, but there is no general agreement about the high-pressure phase diagram.

Several papers have been devoted to the theoretical study of phase transitions in zirconia. In Ref. 8 the rigid-ion model was used and no stable monoclinic structure was found. In the more sophisticated potentialinduced-breathing (PIB) model, ${ }^{9}$ the monoclinic phase is not stable in relation to the fluorite structure, and the tetragonal structure was found to be intrinsically unstable since it has a negative bulk modulus. In our opinion the reason for these failures of ionic models of zirconia lies in their inadequate description of chemical bonding, in which covalency appears to play an important role.

The natural low-pressure low-temperature monoclinic phase of $\mathrm{ZrO}_{2}$ has four formula units per crystalline cell and only four symmetry operations in the point group. Therefore, total-energy $a b$ initio studies of this compound are still very laborious and costly. But even the more symmetrical high-temperature tetragonal (2 formula units, 16 operations) and cubic ( 1 formula unit, 48 operators) phases involve important computations, and (to our knowledge) only one such calculation has been carried out by Jansen and Gardner ${ }^{10}(\mathrm{JG})$. They used the fullpotential linear-augmented-plane-wave (FLAPW) method for studying tetragonal-to-cubic phase transition. This method allows all-electron calculations of the electronic structure of solids to be performed by adopting a localdensity-functional (LDF) approximation for exchange and correlation terms. JG kept lattice parameters for

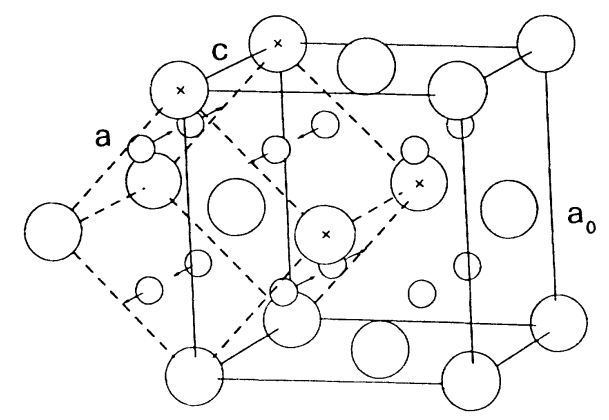

FIG. 1. Geometry of cubic and tetragonal phases of zirconia: The two crystal cells are represented by light solid and heavy dashed lines, respectively. Oxygen and zirconium atoms are represented as small and large circles, respectively. In the tetragonal phase, oxygen atoms can move away from the central positions along the $c$ axis; the displacement is represented by arrows of length $c d_{z}$. Small crosses mark the four zirconium atoms at the corners of the rectangle of the density maps of Fig. 5. 
tetragonal zirconia fixed at their experimental value ${ }^{11}$ $(a=3.64 \AA, c=5.27 \AA$ ), but pairs of oxygens were allowed to move along the $z$ axis. The position $z_{0}$ of the oxygen atoms along the $z$ axis may be described by the adimensional $d_{z}$ parameter: $z_{0}=\left(0.25 \pm d_{z}\right) c$. It was found that the central position of the oxygen atoms $\left(d_{z}=0\right)$ (corresponding to an intermediate structure between cubic and tetragonal phases, space group $\mathrm{P4}_{2} / \mathrm{mmc}$ ) is not stable, while there are two minima on the total-energy-versus $-d_{z}$ curve at $d_{z}=0.052$ [the experimental value for the tetragonal phase is 0.065 (Ref. 6)]. So JG suggested that the cubic structure appears at high temperatures when oxygen atoms can jump between the two mimina. Such a model is in agreement with experimental data for the mean-square atomic displacement of oxygen atoms as a function of temperature. ${ }^{11}$ This value, extrapolated up to the transition temperature of $2400 \mathrm{~K}$, is 0.063 , which practically coincides with the position of the two minima. However, the energy barrier calculated by JG is too low. The estimated transition temperature is $1200 \mathrm{~K}$, in spite of the fact that no account was taken of the possibility that lattice parameter changes might be coordinated with the movement of oxygen atoms. As will be shown later, this coordinated motion plays an important role in the phasetransformation mechanism by lowering the energy barrier for oxygen oscillation between the two minima. Interesting information was also obtained by JG about the electronic structure of the tetragonal phase of zirconia. In particular, electron-charge-density maps were published, and the distribution of electrons was analyzed. The ionization state of oxygen was estimated to be -1.87 . Since it would be -2 for a completely ionic compound, JG were led to hypothesize a certain amount of covalent bonding in tetragonal zirconia.

The present work is similar in spirit to the study by JG in that an $a b$ initio approach is used to investigate the structural stability and electronic structure of the two high-temperature phases of zirconia. The method employed is quite different, however, since a linear combination of atomic orbitals (LCAO), Hartree-Fock (HF) computational scheme was used. ${ }^{12,13}$ The localized basis functions [atomic orbitals (AO's)] are expressed, as usual, as a linear combination of Gaussian-type orbitals (GTO's). ${ }^{14}$ Because of the high number of electrons per unit cell (112 in tetragonal zirconia), it is convenient to get rid of core electrons by adopting well-assessed effective-core-potential (ECP) techniques. ${ }^{15,16}$ The choice of ECP and basis set and some computational problems are discussed in Sec. II. Section III is devoted to the analysis of the structural stability of the two more symmetric phases by considering their HF energy as a function of the involved geometrical parameters. First of all, this study enables information about the most stable configurations and elastic properties of the two phases to be obtained; second, it enables the model proposed by JG for the tetragonal-to-cubic transition to be confirmed, but with different values for the involved parameters; and third, it enables some preliminary speculations about the influence of applied pressure on phase-transition temperatures to be formulated. One may wonder how far the present results might vary if correlation effects were taken into consideration. Well-assessed DF correlation formulas ${ }^{17,18}$ enable us (in principle, at least) to estimate the correlation error quite accurately and to calculate the atomization energy. However, in order to discriminate between two very similar structures, much higher accuracies are required than are possible at present, especially for systems containing heavy atoms such as zirconium treated with an ECP approximation. In Sec. IV we discuss the electronic structure of the calculated equilibrium configurations of cubic and tetragonal zirconia, with reference to band structures, total and projected densities of states (DOS's), Mulliken populations, and electron-density maps. Previous work in this field is comparatively scarce ${ }^{10,19,20}$ We were specially interested in verifying whether or not one can recognize a partially covalent character of bonds in zirconia. The answer is yes, although these concepts are not well defined for compounds such as zirconia, where there is a very large overlap between the AO's of the atoms involved.

\section{BASIS SET AND COMPUTATIONAL DATA}

Selecting a suitable basis set for a crystalline LCAO calculation is often a delicate procedure since the region of functional-parameter space that is accessible (number and character of basis functions) is severely limited by computational limitations. ${ }^{12}$ In the present case, we had the additional problem of adopting suitable ECP's for oxygen and zirconium. The ECP's proposed by the Toulouse group ${ }^{15}$ have recently been the object of a detailed investigation into their performance in crystalline solids. ${ }^{21}$ This investigation confirmed what was already known from molecular studies: Equilibrium geometry, elastic constants, and atomization energy are reproduced within a few percentage points of the corresponding allelectron calculations. The Toulouse ECP's have been adopted for oxygen, but unfortunately they are not available for zirconium. For this atom we resorted to the parameters suggested by Hay and Wadt. ${ }^{16}$ We did this, though no comparable experience exists about the performance of such ECP's in a crystalline environment.

The basis set used for describing valence electrons is shown in Table I. For both oxygen and zirconium a split-valence basis set is used in order to provide sufficient variational freedom: In particular, the oxygen ion must be able to contract in the crystalline field, ${ }^{22}$ and the zirconium orbitals must be able to act as acceptors of electrons back donated by oxygen ions. The basis set for oxygen is a 4-1G set. That is, it consists of two independent sets of $s p$ AO's. The first comes from four contracted GTO's and the second from only one GTO. For the choice of exponents and orbital coefficients, we took as a reference the $5 \mathrm{G}$ valence set proposed by the Toulouse group. ${ }^{23}$ The most diffuse of their five GTO's was taken as an independent function, and its exponent was variationally reoptimized in the case of cubic zirconia, resulting in a slightly more diffuse Gaussian (exponent coefficient 0.212 instead of 0.221 bohr $^{-2}$ ). A $2-1 \mathrm{G}$ set describes $d$ orbitals of zirconium. Optimization of exponents and coefficients was performed for the isolated 
TABLE I. Exponents $\left(\mathrm{bohr}^{-2}\right)$ and coefficients of valence-shell Gaussian functions adopted for the present study. The contraction coefficients multiply individually normalized Gaussions. $y[ \pm z]$ stands for $y \times 10^{ \pm z}$.

\begin{tabular}{lllll}
\hline \hline & $\begin{array}{c}\text { Shell } \\
\text { type }\end{array}$ & Exponents & \multicolumn{2}{c}{ Coefficients } \\
& $s p$ & $4.0[-1]$ & $s$ & $p$ or $d$ \\
\hline Zirconium & $s p$ & $1.6[-1]$ & 1.0 & 1.0 \\
& $d$ & 2.248 & 1.0 & 1.0 \\
& & $7.81[-1]$ & & $7.189[-2]$ \\
& $d$ & $2.396[-1]$ & & $4.975[-1]$ \\
Oxygen & $2.371[+1]$ & $1.694[-2]$ & 2.0 \\
& $s p$ & 6.227 & $-1.615[-1]$ & $1.638[-2]$ \\
& & 2.108 & $1.129[-1]$ & $2.990[-1]$ \\
& & $7.065[-1]$ & $6.700[-1]$ & $4.709[-1]$ \\
& & $2.12[-1]$ & 1.0 & 1.0 \\
\hline
\end{tabular}

$\mathrm{Zr}^{2+}$ ion $\left(d^{2}\right.$ configuration). Determination of $s p$ AO's for zirconium was much more delicate because of their diffuse character. The final choice reproduced in Table I is a compromise between satisfactory variational performance and computational problems. It might be argued that the choice of the basis set for zirconium is not critical, especially as far as $5 p$ functions are concerned. These AO's are unoccupied in the isolated atom, and they are further destabilized by the crystalline field; so they should not contribute to the ground-state wave function. However, as we shall discuss below, the participation of those AO's in the occupied bands is not negligible, and one may wonder if the set here employed is adequate. The $1-1 \mathrm{G} s p$ valence basis set reported in Table $I$ is certainly unable to reproduce the corresponding orbitals of the isolated atom, which have much larger tails. For instance, in the $5 s$ AO of zirconium, as described by Hay and Wadt $^{16}$ with three contracted GTO's, about half of the electron charge is outside a sphere of a 4-bohr radius, corresponding approximately to a nearest-neighbor $\mathrm{Zr}-\mathrm{O}$ distance, while this fraction is negligible with the present basis set. Inclusion of very diffuse functions for describing the outer part of the AO's is impossible in practice, because it would increase both the cost of the calculation (the number of integrals to be computed and stored may become enormous) and the risk of catastrophes due to pseudolinear dependence. ${ }^{12}$ On the other hand, in a crystalline environment, basis-set deficiencies are largely compensated for by the possibility of exploitation of the basis functions on neighboring atoms. Consider, for instance, the case of an isolated $\mathrm{Zr}^{2+}$ ion in the $(5 s)^{2}$ configuration (inner shells are treated with a standard STO-6G basis set). If our $1-1 \mathrm{G}$ set is used for valence electrons, the total-energy and valence $5 s$ levels are 3515.7814 and -0.6938 hartree, respectively. These figures change to 3515.8979 and -0.7396 if we add to our $1-1 G$ set the Hay-Wadt $3 \mathrm{G}$ set, ${ }^{16}$ which shows the importance of the latter function in describing the outer part of the valence-electron distribution. Instead, when we supplement the 1-1G set with "ghost" $s p$ functions centered at the location of neighboring oxygen atoms in cubic zirconia (exponents and coefficients are as for oxygens in Table I), then the above figures become -3516.0381 and
-0.7001 . In a variational sense, these data justify the use of the basis set here proposed. However, the interpretation of results in chemical terms requires a careful analysis (see Sec. IV), and in the evaluation of atomization energy one must account for an important correction related to the so-called basis-set-superposition error (BSSE). ${ }^{24}$

Finally, in Table II, we give some data on the cost of the present computations. One may note that they include the case of monoclinic zirconia, which has been the object of a single test calculation. At the present time, a systematic structural investigation of this phase, though feasible in principle, is too expensive.

\section{TOTAL ENERGY AND RELATED PROPERTIES}

The structural properties of cubic zirconia may be investigated by calculating the total energy as a function of the lattice parameter $a_{0}$, while for the tetragonal phase one must consider the three geometrical parameters $a, c$, and $d_{z}$ (see Fig. 1). Even if the cubic structure is unstable at zero temperature, it corresponds to a relative minimum with respect to deformations, which preserve oxygen atoms in their central position $\left(d_{z}=0\right)$.

Therefore, a preliminary series of computations was devoted to the cubic phase, its equilibrium geometry, and elastic properties. The calculated lattice parameter $a_{0}$ $(5.035 \AA)$ agrees with experimental findings [5.086 $\AA$ (Ref. 5)] to within $1 \%$. The comparison of calculated elastic properties with available experimental and theoretical data is provided in Table III. Fitting calculated-energy-versus-volume data into the Murnaghan equation of $\operatorname{stat}^{25}$ gives a value of bulk modulus $B=222 \mathrm{GPa}$. In each case, in order to obtain the elastic constants, seven points of the totalenergy-versus-deformation curve were calculated in the interval -0.02 to +0.02 of the deformation parameter, and polynomial fitting was employed. The value of $C_{11}-C_{12}$ was obtained by performing tetragonal distortions of the cubic unit cell with oxygen atoms fixed at the central position. Using the equation $\left(C_{11}+2 C_{22}\right) / 3=B$ and the previously obtained bulk modulus, separate values for $C_{11}$ and $C_{22}$ were calculated. The $C_{44}$ elastic 
TABLE II. Computational data for zirconia. All calculations refer to the experimental geometries; ECP's and the basis set are as described in Sec. II. "Good" tolerances have been employed for the truncation parameters (Ref. 12).

\begin{tabular}{|c|c|c|c|}
\hline Crystal structure & Cubic & Tetragonal & Monoclinic \\
\hline \multicolumn{4}{|l|}{ Structural data } \\
\hline Atoms/cell & 3 & 6 & 12 \\
\hline $\mathrm{AO} /$ cell & 34 & 68 & 136 \\
\hline Point-group operators & 48 & 16 & 4 \\
\hline \multicolumn{4}{|l|}{$\begin{array}{l}\text { Number of two-electron } \\
\text { integrals }\left(\times 10^{6}\right)\end{array}$} \\
\hline Coulomb & 213 & 653 & 1077 \\
\hline Exchange & 175 & 557 & 1004 \\
\hline \multicolumn{4}{|l|}{$\begin{array}{l}\text { Computational times } \\
\text { (IBM } 3090 / 170 \text { s) }\end{array}$} \\
\hline Two-electron integrals & 754 & 2414 & 7309 \\
\hline One-electron integrals & 56 & 123 & 501 \\
\hline Self-consistent-field procedure & 23 & 240 & 1250 \\
\hline
\end{tabular}

constant was obtained by rhombohedral deformation of the unit cell. The results show that the calculated bulk modulus is in good agreement with the experimental data, ${ }^{26}$ while there is definite disagreement for other elastic constants, especially $C_{12}$. The elastic constants of ionic crystals obtained through all-electron and ECP-HFLCAO techniques are usually close to the experimental values when good basis sets are used. ${ }^{27}$ The present disagreement is certainly to be attributed in part to basis-set inadequacies, in part to uncertainties in the measured data (obtained on $\mathrm{Y}_{2} \mathrm{O}_{3}$-stabilized zirconia and extrapolated to the zero-impurity-concentration $\operatorname{limit}^{26}$ ), and in part to the instability of the cubic phase at zero temperature.

As the next step of our study, we tried to optimize the geometry of the tetragonal phase. Instead of considering the three structural parameters on the same footing, we decided to study the dependence of energy on $a$ and $c$ at two selected planes $d_{z}=$ const. The first value $\left(d_{z}= \pm 0.065\right)$ corresponds to the experimentally measured shifts of oxygen atoms from their central positions in tetragonal zirconia; the second one $\left(d_{z}=0\right)$ identifies the plane containing the cubic structure. The two planes are referred to conventionally in the following as the

TABLE III. Calculated and experimental determinations of elastic properties of cubic zirconia. All data are in GPa. The rigid-ion (RI) and potential-induced-breathing (PIB) models are discussed in the Introduction. Experimental data were obtained by extrapolation of data measured on $\mathrm{Y}_{2} \mathrm{O}_{3}$-stabilized zirconia to the zero-impurity-concentration limit.

\begin{tabular}{lcrrr}
\hline \hline & Present calc. & RI $^{\mathrm{a}}$ & PIB $^{\mathrm{b}}$ & Experiment $^{\mathrm{c}}$ \\
\hline$B$ & 222 & 115 & 288 & 194 \\
$C_{11}$ & 628 & 222 & 560 & 417 \\
$C_{12}$ & 19 & 61 & 153 & 82 \\
$C_{44}$ & 82 & 54 & 180 & 47 \\
\hline \hline
\end{tabular}

${ }^{\text {a }}$ Reference 8 .

${ }^{\mathrm{b}}$ Reference 9.

${ }^{\mathrm{c}}$ Reference 26. "tetragonal" and "cubic" planes, respectively. On the two planes the other two parameters were independently changed in the range $3.43<a<3.68 \AA$ and $4.84<c<5.38 \AA$. Sixteen energy points were calculated at each plane and fitted by a second-degree polynomial. The coefficients and corresponding points of minimal energy of this tetragonal and cubic energy surfaces are listed in Table IV. For both tetragonal and cubic zirconia, the root-mean-square deviations of calculated from fitted energy values are less than $2.5 \times 10^{-4}$ hartree, while the energy interval spanned by calculated values is $10^{-2}$ hartree. Inclusion of third-power terms in the fitting polynomial does not improve significantly the quality of fitting. This is not surprising, since the numerical noise of the present calculation (associated essentially with tolerances adopted for the truncation of infinite lattice sums) is of the same order of magnitude as the average deviation quoted above; it may be of interest to note that the reported numerical error for the FLAPW calculation is similar $\left(5 \times 10^{-4}\right.$ hartree $)$. From Table IV one can note that the values of lattice parameters for the cubic plane obtained by this fitting differ slightly from those previous-

TABLE IV. Coefficients of the second-degree polynomial $E(a, c)=A c^{2}+B c a+C a^{2}+D c+E a+F$, representing the dependence of total energy (hartree) per $\mathrm{ZrO}_{2}$ formula unit on the lattice parameters (angstroms) for "cubic" and "tetragonal" phases (see explanation in the text). $y[ \pm z]$ stands for $y \times 10^{ \pm z}$. The $a, c$, and $E$ values, corresponding to the minima, are also shown in the same units.

\begin{tabular}{lcc}
\hline \hline & Cubic & Tetragonal \\
\hline$A$ & $9.97[-2]$ & $6.11[-2]$ \\
$B$ & $1.89[-2]$ & $3.11[-2]$ \\
$C$ & $3.55[-1]$ & $3.37[-1]$ \\
$D$ & -1.07 & $-7.48[-1]$ \\
$E$ & -2.62 & -2.55 \\
$F$ & 7.37 & 5.87 \\
$a$ & 3.561 & 3.558 \\
$c$ & 5.040 & 5.258 \\
$E$ & -34.5908 & -34.5981 \\
\hline \hline
\end{tabular}


ly reported (note, however, that the $c / a$ ratio is 1.415 , that is, very close to the theoretical value $2^{1 / 2}$ ). This discrepancy is due to the different fitting techniques employed in the two cases. For instance, use of seconddegree fitting in $a$ and $c$ gives a value of $236 \mathrm{GPa}$ for the bulk modulus of the cubic phase, which is by $6.3 \%$ larger than obtained by the much more accurate onedimensional fitting with the Murnaghan equation. Concerning the tetragonal phase, the values obtained for the structural parameters $(a=3.558 \AA, c=5.258 \AA)$ are in reasonable agreement with experimental data.

For a complete structural investigation of the tetragonal phase, the dependence of energy on the $d_{z}$ parameter should be analyzed. A complete study of this kind is too expensive. However, we checked that in the explored area of $a$ and $c$ parameters the equilibrium positions. of oxygens lie in interval $0.05<\left|d_{z}\right|<0.08$. So our representation of tetragonal zirconia with oxygen positions fixed at $d_{z}=0.065$ is not far from the optimized geometry. In particular, the experimental finding is confirmed that the tetragonal phase is more stable than the cubic one at low temperatures.

The dependence of energy on $d_{z}$ was investigated more closely for the $(a, c)$ equilibrium values of tetragonal and cubic zirconia. The corresponding curves are shown in Fig. 2. For comparison, the results obtained by $\mathrm{JG}$ in their study of the influence of the $d_{z}$ parameter are also reproduced (dotted line). For the $a=3.558 \AA$ and $c=5.258 \AA$ values, fitting of calculated points with a fourth-power polynonmial provides the location of the energy minima: $d_{z}= \pm 0.076$. This was considered as the equilibrium geometry for the tetragonal phase, and electronic structure data for this geometry are reported in the next section.

The total-energy curves of Fig. 2 represent a doublewell structure. We shall consider the same model of

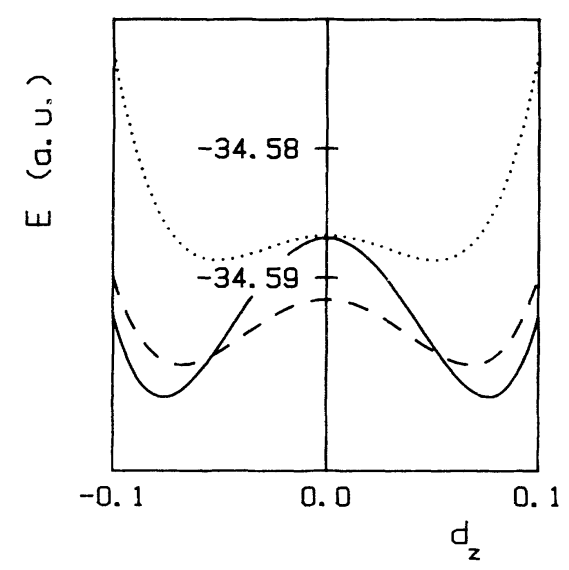

FIG. 2. Dependence of energy on $d_{z}$, the fractional displacement of oxygen atoms (see Fig. 1), for different values of the lattice parameters $a$ and $c$. In this figure and the following two, a.u. stands for atomic units. "tetragonal" zirconia $(a=3.558 \AA$, $c=5.258 \AA$ ) (solid curve), "cubic" zirconia $(a=3.561 \AA$, $\left.c=a 2^{1 / 2}\right)$ (dashed curve), and JG results (Ref. 15) $(a=3.64 \AA$, $c=5.27 \AA$ ) (dotted curve): the energy has been shifted to coincide with the present "cubic" result at $d_{z}=0$. tetragonal-to-cubic phase transition as suggested by JG. The cubic phase appears at high temperature when the system has the possibility of crossing almost freely the energetic barrier between the two tetragonal structures. To obtain the correct value of this barrier, we must compare totally optimized energies of tetragonal and cubic structures, because the shift of oxygens to their central positions leads to considerable changes in lattice constants (this fact was not taken into account by JG). The calculated activation barrier is 0.0075 hartree, corresponding to a phase-transition temperature of $2300 \mathrm{~K}$, in good agreement with the experimental value.

As well as the temperature, the pressure $P$ can be a driving force for phase transitions. In order to estimate the influence of pressure on the phase transformation, one must calculate the free energy $(F=E+P V)$. Comparison of free-energy minima at different pressures on the tetragonal and cubic planes allows us to estimate the transition temperature as a function of pressure according to the model discussed above. We have found that up to $50 \mathrm{GPa}$ the energy barrier remains approximately constant, and the temperature needed to activate the phase transition lies in interval $2000-3000 \mathrm{~K}$. This means that the line dividing cubic and tetragonal phase regions on the $\mathrm{ZrO}_{2}$ phase diagram goes almost parallel to the pressure axis up to $50-\mathrm{GPa}$ values. This conclusion is in agreement with experimental data about the highpressure low-temperature part of zirconia phase diagram, where no evidence was found for cubic-phase presence. However, these estimates must be taken with caution, because of the relatively large numerical error that affects calculated data.

It may be of interest to compare the calculated atomization energy of zirconia to the experimental data. JG have estimated the latter to be 0.84 hartree per formula unit. By subtracting from the energies reported in Table IV the energies for the atomic species treated in the same approximation and with the same basis set $(-15.658$ and -2.329 hartree for oxygen and zirconium, respectively), we obtain an atomization energy of 0.946 and 0.953 hartree for the cubic and tetragonal phases, respectively. Incidentally, we can observe that the results obtained from the unique calculation effected for monoclinic zirconia at the experimental geometry (see Table II) would give an atomization energy of 0.951. Since no geometricalparameter optimization was effected in this case, it is easy to predict that a slightly higher stability would be found for monoclinic zirconia with respect to the other phases, in agreement with the experiment. All calculated stabilities appear too high, even because to these data one must add the correlation contribution. This excess energy is clearly attributable to the uncorrect reference atomic energies, which do not include any correction for the BSSE. ${ }^{24}$ An empirical recipe for obtaining more reliable reference atomic energies, which has given satisfactory results in a number of crystalline calculations, is as follows. The isolated atom calculations are performed with the same basis set as used in the crystalline calculation, but substituting the outermost $s p$ shell with two independent single-Gaussian $s p$ shells whose exponents are variationally optimized. In the present case the reference 
atomic energies decrease to -15.660 for oxygen (outer exponents 0.22 and 0.76 bohrs $^{-2}$ ) and to -2.709 for zirconium (outer exponents 0.38 and 0.040 bohrs $^{-2}$ ). As anticipated in Sec. II, the energy gain is extremely important for zirconium and probably overestimated, but is indicative of the importance of BSSE's with poor basis sets. The corresponding calculated binding energy would decrease to 0.578 a.u. for tetragonal zirconia.

As stated in the Introduction, no attempt has been made to estimate the effect of correlation corrections on the relative stability and equilibrium geometry of the two structures. Accurate exploratory work is needed before extending the use of correlation functionals to such subtle applications, especially when systems are concerned where transition-metal atoms are present.

\section{ELECTRONIC STRUCTURE}

In this section we describe the electronic structure of the cubic and tetragonal phase of zirconia calculated at their theoretical equilibrium configurations. Figure 3 reports band structures, and Fig. 4 total and projected densities of states. Reference will also be made in the following to the Mulliken population analysis data given in Table V.

The band structures for the two phases are similar, exhibiting (from lower to higher energies) a narrow valence band, essentially associated with $s$ orbitals of oxygen, a wider valence band, primarily from $p$ AO's of oxygen (small but important contributions from zirconium to these bands will be discussed in the following), and a con-
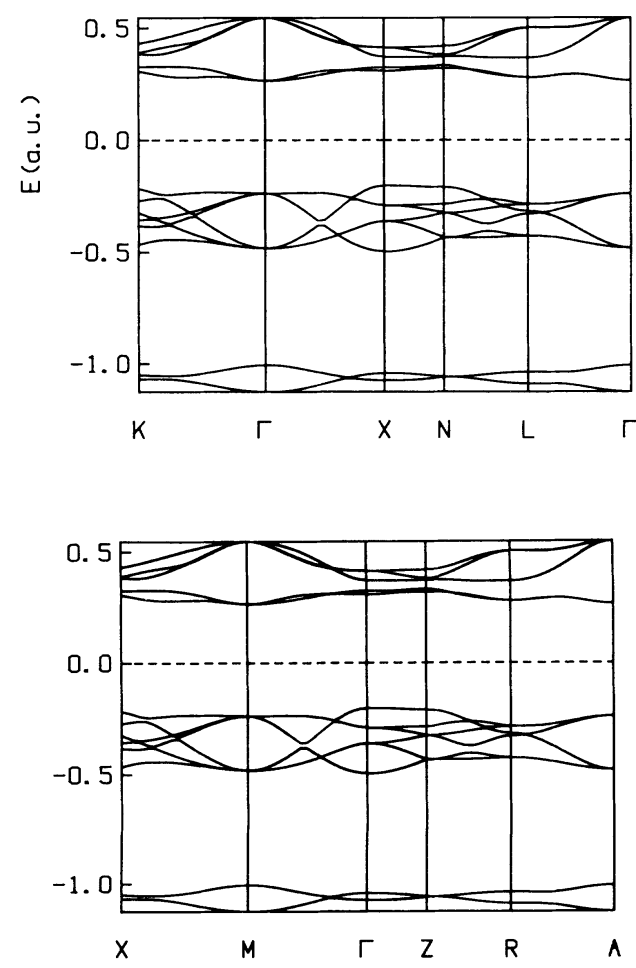

FIG. 3. Band structure for cubic (top) and tetragonal (bottom) zirconia at their calculated equilibrium configurations.
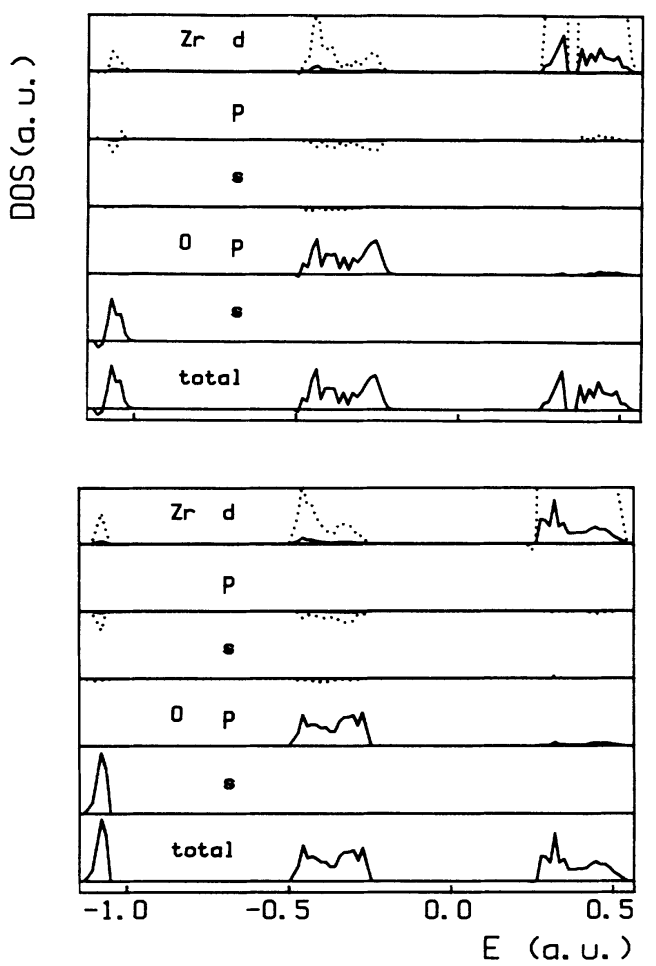

FIG. 4. Total and projected DOS's for cubic (top) and tetragonal (bottom) zirconia at their calculated equilibrium configurations. Contributions from different types of AO's are attributed owing to a Mulliken population analysis. Dotted DOS's are multiplied by a factor 10 .

duction band, coming from $4 d$ AO's of zirconium. The valence portion of this structure is typical of ionic oxides. The analysis of a large number of HF calculations has shown that the bandwidth of the $p_{0}$ band is strongly correlated to the distance $D_{\mathrm{O}-\mathrm{O}}$ between the nearest oxy-

TABLE V. Mulliken population analysis for cubic and tetragonal zirconia. For tetragonal zirconia, the $p$-oxygen population is $\left(p_{x}+p_{y}+p_{z}\right) / 3$, and the two overlap populations refer to $\mathrm{Zr}-\mathrm{O}$ neighbors in the first coordination sphere at the indicated distance $d$.

\begin{tabular}{lcc}
\hline \hline Crystal structure & Cubic & Tetragonal \\
\hline Gross charges & & \\
Zirconium & 0.679 & 0.715 \\
$s$ & -0.088 & -0.125 \\
$p_{x}=p_{y}$ & -0.131 & -0.152 \\
$p_{z}$ & -0.131 & -0.103 \\
$d_{z^{2}}$ & 0.098 & 0.140 \\
$d_{x^{2}-y^{2}}$ & 0.098 & 0.367 \\
$d_{z y}=d_{x z}$ & 0.322 & 0.287 \\
$d_{x y}$ & 0.322 & 0.165 \\
Oxygen & 7.661 & 7.642 \\
$s$ & 1.973 & 1.988 \\
$p$ & 1.896 & 1.885 \\
Overlap populations & & \\
$(\mathrm{Zr}-\mathrm{O})[d(\AA)]$ & $0.005[2.180]$ & $-0.010[2.000]$ \\
$(\mathrm{Zr}-\mathrm{O})[d(\AA)]$ & & $-0.001[2.470]$ \\
$(\mathrm{O}-\mathrm{O})[d(\AA)]$ & $-0.063[2.517]$ & $-0.036[2.629]$ \\
\hline \hline
\end{tabular}


gen atoms and, to a lesser extent, to their number. ${ }^{28}$ Even in the present case, the appreciable difference between the two bandwidths $(7.14 \mathrm{eV}$ for tetragonal and $7.97 \mathrm{eV}$ for cubic zirconia) may be attributed to the different $D_{\mathrm{O}-\mathrm{O}}$ values in the two equilibrium structures (2.63 and $2.52 \AA$, respectively). On the other hand, these bandwidths are smaller, by about $1 \mathrm{eV}$, than those of purely ionic compounds (such as $\mathrm{BeO}$ or bcc $\mathrm{MgO}$ ), having comparable $D_{\mathrm{O}-\mathrm{O}}$ values. This fact is indicative of a less marked ionic character of this compound. The pattern here described is qualitatively similar to that reported by Evarestov, Leko, and Sokolov ${ }^{19}$ and Zandiehnadem and Murray, ${ }^{20}$ who calculated band structures and DOS's of the cubic, tetragonal, and monoclinic phases, using a complete-neglect-of-differential-overlap (CNDO) approximation and an $a b$ initio LDF-LCAO approach, respectively. In particular, the present valence bands reproduce almost exactly those of Ref. 20, if the energy scale of the latter ones is contracted by a factor of 1.5. The difference is more important in the evaluation of the gap between valence and conduction bands (optical gap). For the cubic and tetragonal phases, the above-mentioned authors obtain 3.84 and $4.11 \mathrm{eV}$, compared with the present values of 12.3 and $13.3 \mathrm{eV}$, respectively, and with a "true" value, which is estimated to be about $6 \mathrm{eV} .{ }^{20}$ It is well known that optical gaps are systematically underestimated by LDF and overestimated by HF calculations. Here the relatively poor description of virtual bands, as a result of basis-set inadequacies, contributes to increasing the separation between valence and conduction bands. The CNDO approximation ${ }^{19}$ exaggerates bandwidths, as usual, but provides a fairly accurate estimate of the optical gap.

Now consider the projected DOS's of Fig. 4 and, in particular, the contribution of $\mathrm{Zr}$ AO's to the valence bands. Since the two schemes are similar, we shall concentrate our attention on the case of cubic zirconia. $d$ function contributions are more important in the $p_{\mathrm{O}}$ band, but are not negligible in the $s_{O}$ band. On the whole, the Mulliken population of zirconium $d$ AO's is 1.16 (1.25 for tetragonal zirconia). Zirconium $p$ and $s$ AO's contribute to some extent to valence bands, but as is evident from the plots, their population is negative. This result is paradoxical in the frame of standard Mulliken analysis (see Table V): To the valence shell of zirconium, we should attribute 0.68 electrons, resulting from $1.16 d$ electrons minus $0.48 s p$ electrons. In order to clarify this point, it is useful to consider the zirconium atom in its crystalline environment by making reference to ligand-field theory in the strong-field limit (see, for instance, Ref. 29). In the cubic environment of the nearest-neighbor negative oxygen ions $\left(O_{h}\right.$ point-group symmetry), atomic levels of zirconium are shifted up in energy and partially resolved according to symmetry properties. They may combine with states of the same symmetry resulting from $s$ and $p \sigma$ AO's on oxygens (which generate states of $A_{1 g}, T_{2 g}, A_{2 u}, T_{1 u}$ symmetry) and from $p \pi$ AO's on the same atoms $\left(E_{g}, T_{1 g}, T_{2 g}, E_{u}, T_{1 u}, T_{2 u}\right) . d$ levels split into a group of two $E_{g}$ levels and three $T_{2 g}$ levels. The former group lies significantly lower in energy with respect to the latter, as a consequence of the more favorable spatial disposition with respect to the negative centers. This fact is apparent from the band structure, where the two $d-E_{g}$ conduction bands lie lower in energy with respect to the three $d-T_{2 g}$ conduction bands (in cubic zirconia, there is even a small gap between the two groups). As far as concerns the ability to form bonds with AO's on oxygen atoms, both spatial disposition and symmetry favor $d-T_{2 g}$ orbitals. This is why their contribution to valence bands is more than 3 times that of $d-E_{g}$ AO's. An analogous situation is maintained in the presence of the tetragonal distortion in spite of some significant changes. Now consider the problem of negative-valence $p$ and $s \mathrm{Zr} \mathrm{AO}$ populations. These AO's (which belong to the $A_{1 g}$ and $T_{1 u}$ irreducible representations of the $O_{h}$ point group, respectively) are much more diffuse than $4 d$ AO's and overlap considerably with nearest-neighbor oxygens (see Sec. II). The presence of the negative cage of oxygens has the effect of further increasing their spatial extent (a fraction of electrons migrate outside the cage). This is the counterpart of the well-known effect of anion contraction in ionic solids. $^{22}$ Owing to their spatial disposition, $p$ AO's are destabilized to a lesser extent than $s$ functions and because of their symmetry, are more likely to contribute to bonding crystalline orbitals associated with oxygens. On the whole, we would expect small but non-negligible contributions of $s$ and, especially, $p$ AO's to the occupied manifold of crystalline states. The reason why the outcome of the Mulliken analysis is negative $s p \mathrm{Zr}$ populations is a consequence of the fact that functions centered on oxygen atoms make an essential contribution to the description of the tail of valence $s p$ AO's of zirconium, as discussed in Sec. II. We can formally describe one such orbital as $Z=\alpha A+\beta B+\gamma C$, where $A$ and $B$ are normalized Gaussian functions centered on zirconium, $C$ is a normalized group orbital centered on oxygens, and $\alpha, \beta$, $\gamma$ are shape coefficients. In order to describe a valence $s$ or $p \mathrm{AO}$ in the crystalline environment with maximum density near the oxygen atoms, $\alpha$ and $\beta$ should be smaller in absolute value and of opposite sign with respect to $\gamma$. When a Mulliken analysis is performed, the $C$ function is attributed to oxygens. Therefore, the net population on $\mathrm{Zr}$ is resolved with a small (positive) contribution from $A$ and $B$ which is attributed to zirconium, a relatively much larger (and positive) contribution from $C$ which is attributed to oxygens, and with a sizable (and negative, because of the sign of the coefficients) "overlap population" which is equally shared between the two atomic species. The risks of a mechanical application of standard Mulliken analysis is particularly evident when one considers zirconium-oxygen overlap populations in tetragonal zirconia. One would expect the two bonds, in particular the short one, to be partially covalent: If so, they should be characterized by positive overlap populations, while the opposite is found (see Table III).

The above discussion of the Mulliken analysis data finds a confirmation in the results obtained using a molecular model of tetragonal zirconia, recently proposed by Sautet. $^{30}$ According to this model, zirconium is surrounded by four hydroxyl groups $(\mathrm{Zr}-\mathrm{OH})$, representing the short $\mathrm{Zr}-\mathrm{O}$ bonds, and four water molecules 
$\left(\mathrm{Zr}-\mathrm{OH}_{2}\right)$, corresponding to the long bonds. This model is surprisingly effective in predicting the equilibrium geometry of the first coordination sphere around zirconium and the electronic structure of that atom in the crystalline environment and can be used therefore in catalytic studies. When one describes this molecular complex with the same basis set for zirconium and oxygen as here employed and adopts the bulk-optimized geometry for oxygen atoms around zirconium, the Mulliken populations are similar to those shown in Table V. In particular, one finds negative $s \mathrm{Zr}$ and $p \mathrm{Zr}$ populations, near-zero overlap population for the long $\mathrm{Zr}-\mathrm{O}$ bond, and a slightly positive one for the short bond. However, when one uses the Hay-Wadt basis set for zirconium (that is, a reasonable description of those AO's, the picture is completely altered. Both $s$ and $p \mathrm{Zr}$ populations become positive (about 0.4 electrons on these AO's), and the $d$ population is also increased slightly; the total Mulliken charge on zirconium is $1.85 e$. The overlap populations of the short and long bonds become 0.24 and 0.04 electrons, respectively.

This discussion reveals the limits of the Mulliken analysis in critical situations when the overlap between AO's from different atoms is very large and when it is not possible, because of computational problems, to use basis function which are attributable properly to a given atomic species. No attempt has been made here to correct this false result by projecting the density matrix onto a minimal basis set of AO's, as is sometimes done in molecular calculations. However, the present discussion shows
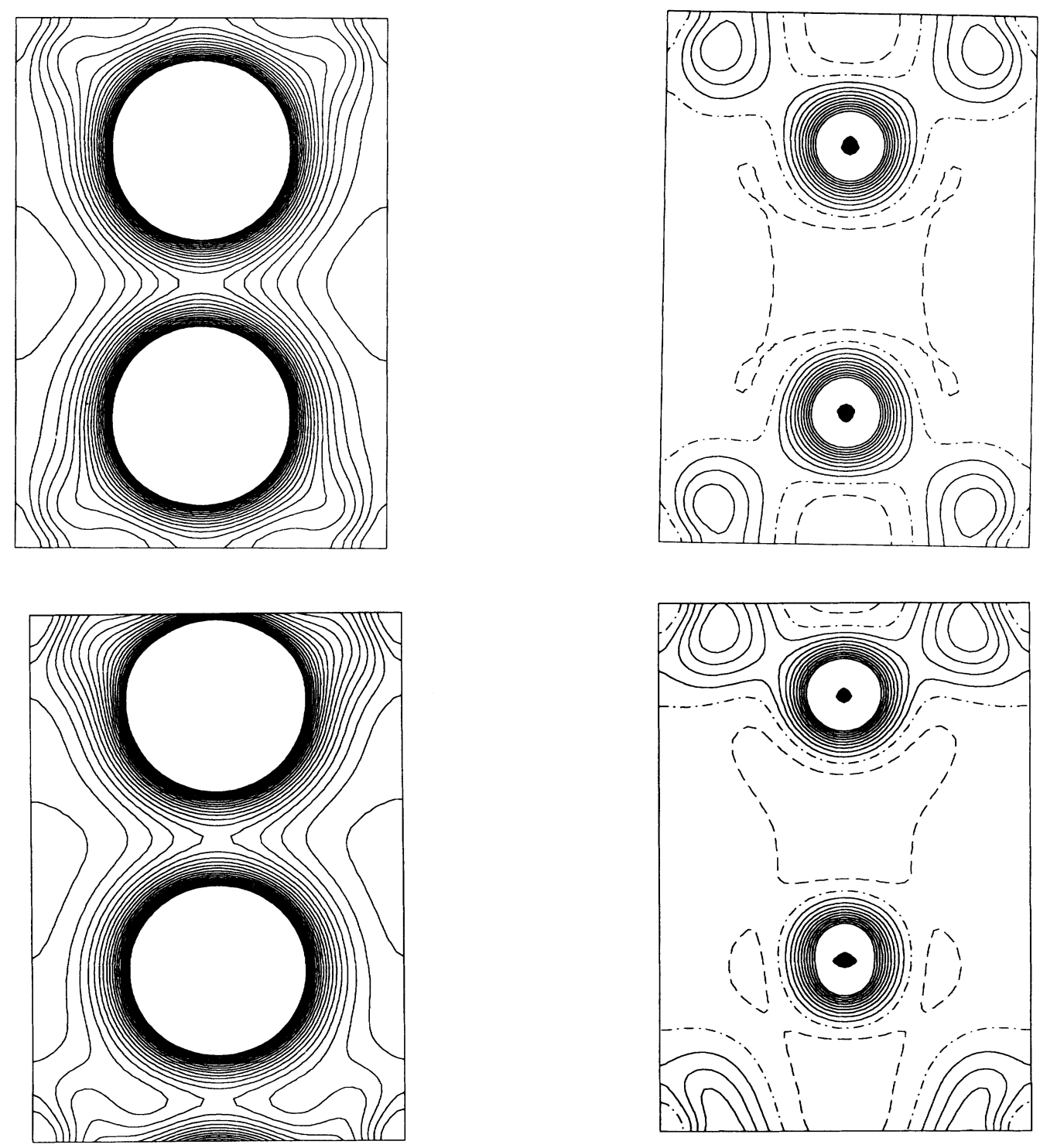

FIG. 5. Total and difference valence-charge-density maps for cubic (top) and tetragonal (bottom) zirconia at their calculated equilibrium configurations. Difference maps (on the right) are evaluated with reference to a superposition of electron densities from isolated $\mathrm{O}^{2-}$ ions (no valence electrons on $\mathrm{Zr}^{4+}$ ). The rectangle of the plot is as shown in Fig. 1. The distance between isodensity curves is $0.005 e / \mathrm{bohr}^{3}$. 
that a non-negligible positive population $(0.2-0.4$ electron) should be attributed to sp AO's on zirconium so that this atom can be assigned a valence charge of about 1.5 electrons. That is, the covalent character of zirconia seems far more pronounced than is proposed by JG.

Finally, let us briefly discuss the overlap populations between the nearest oxygen atoms, which are also given in Table $\mathrm{V}$, with the present basis set. We can consider them as more meaningful than the ones involving zirconium. The antibonding character of the O-O interaction is revealed by the appreciably negative values on their overlap populations, much more markedly so in the case of cubic zirconia. McCullough and Trueblood ${ }^{31}$ have attributed the primary reason for the instability of cubic zirconia to the uncommonly short distance $(2.53 \AA$ ) between each oxide ion and its six nearest oxygens (in tetragonal and monoclinic zirconia, the O-O distances within a given zirconium coordination polyhedron have an average value of 2.64 and $2.75 \AA$, respectively). The present results might support their interpretation.

The density maps of Fig. 5 provide further insight into the electronic structure of the two phases. With respect to the all-electron maps of $\mathrm{JG}$, the present valence-only densities reveal the complicated character of the electronic structure in a neighborhood of zirconium nuclei, and this confirms the partially covalent character of the $\mathrm{Zr}$ $\mathrm{O}$ bonds - the difference between short and long $\mathrm{Zr}-\mathrm{O}$ bonds in tetragonal zirconia is particularly impressive. On the other hand, there is almost perfect quantitative agreement between JG's and our calculated electron density at the midpoint of the $\mathrm{O}-\mathrm{O}$ bond, where the contribution from core electrons is negligible. These features are even more evident in the difference maps (valence minus superposition of isolated $\mathrm{O}^{2-}$ ions). The presence of $d \mathrm{Zr}$ electrons appears to be most important along the short $\mathrm{Zr}-\mathrm{O}$ bond in tetragonal zirconia. The contribution from sp AO's is not apparent because of their diffuse character. The other important feature that emerges from these plots is the contraction of anions which causes an accumulation of electrons near the oxygen nuclei with respect to isolated ions. ${ }^{22}$

\section{PROSPECTIVE WORK}

The present work is intended as a preliminary step in a long-term investigation concerning the surface and catalytic properties of zirconia. A slab model will be used for simulating different exposed crystal faces. For the rest the computational techniques to be applied will be the same as here employed. An essential prerequisite for such a study to provide reliable data is the correct description of the corresponding bulk structure. We believe the results presented here are encouraging, as far as the structural and electronic properties of cubic and tetragonal zirconia are concerned. The surface studies to be undertaken will concern the latter structure, which is known to be the prevailing one in microcrystalline zirconia catalysts. A parallel investigation into bulk monoclinic zirconia is envisaged in the near future.

\section{ACKNOWLEDGMENTS}

Financial support by CSI Piemonte, the Italian Ministero della Ricerca Scientfica (MURST), and the Italian CNR (Progetto finalizzato: Materiali speciali per tecnologie avanzate) is gratefully acknowledged. Thanks are due to $\mathrm{E}$. Aprà for help in computational work and to $\mathbf{P h}$. Sautet for useful discussions. One of us (E.S.) is indebted to the University of Turin for partial support.
${ }^{1}$ C. Morterra, R. Aschieri, V. Bolis, B. Fubini, and M. Volante, Gazz. Chim. Ital. 118, 479 (1988).

${ }^{2}$ Zirconia '88. Advances in Zirconia Science and Technology, edited by S. Meriani (Elsevier, New York, 1989).

${ }^{3}$ Physica B + C 150 (1988).

${ }^{4} \mathrm{R}$. Stevens, in Ceramic Monographs-Handbook of Ceramics (Verlag Schmid GmbH, Freiburg, 1985).

${ }^{5}$ C. J. Howard, R. J. Hill, and B. E. Reichert, Acta Crystallogr. B 44, 116 (1988).

${ }^{6} \mathrm{G}$. Teufer, Acta Crystallogr. 15, 1187 (1962).

${ }^{7}$ R. W. G. Wyckoff, Crystal Structures, 2nd ed. (Wiley, New York, 1963), Vol. 1.

${ }^{8}$ L. L. Boyer and B. M. Klein, J. Am. Ceram. Soc. 68, 278 (1985).

${ }^{9}$ R. E. Cohen, M. J. Mehl, and L. L. Boyer, Physica B + C 150, 1 (1988).

${ }^{10}$ H. J. F. Jansen and J. A. Gardner, Physica B+C 150, 10 (1988).

${ }^{11}$ P. Aldebert and J. P. Traverse, J. Am. Ceram. Soc. 68, 34 (1985).

${ }^{12}$ C. Pisani, R. Dovesi, and C. Roetti, Hartree-Fock ab initio Treatment of Crystalline Systems, Lecture Notes in Chemistry, Vol. 48 (Springer-Verlag, Heidelberg, 1988).
${ }^{13}$ R. Dovesi, C. Pisani, C. Roetti, M. Causà, and V. R. Saunders, CRYSTAL 88. Program n.577, Quantum Chemistry Program Exchange, Indiana University, Bloomington, IN, 1989. From Phys. Rev. B 44, 3509 (1991) Ref. 22.

${ }^{14}$ See, for instance, W. J. Hehre, R. Ditchfield, R. F. Stewart, and J. A. Pople, J. Chem. Phys. 52, 2769 (1970).

${ }^{15}$ P. H. Durand and J. C. Barthelat, Theor. Chim. Acta 38, 283 (1975).

${ }^{16}$ P. J. Hay and W. R. Wadt, J. Chem. Phys. 82, 270 (1985).

${ }^{17}$ R. Colle and O. Salvetti, Theor. Chim. Acta 37, 329 (1975); 53, 55 (1979); R. Colle, A. Fortunelli, and O. Salvetti, J. Chem. Phys. 80, 2654 (1984); M. Causà, R. Dovesi, C. Pisani, R. Colle, and A. Fortunelli, Phys. Rev. B 36, 891 (1987); M. Causà, R. Colle, A. Fortunelli, R. Dovesi, and C. Pisani, Phys. Scr. 38, 194 (1988).

18J. P. Perdew, Phys. Rev. B 33, 8822 (1986); 34, 7406 (1986).

${ }^{19}$ R. A. Evarestov, A. V. Leko, and A. R. Sokolov, Dokl. Akad. Nauk SSSR 299, 1185 (1988).

${ }^{20}$ F. Zandiehnadem and R. A. Murray, Physica B+C 150, 19 (1988).

${ }^{21}$ M. Causà, R. Dovesi, and C. Roetti, Phys. Rev. B 43, 11937 (1991).

${ }^{22}$ P. W. Fowler and P. Tole, Surf. Sci. 197, 457 (1988). 
${ }^{23}$ Y. Bouteiller et al. (private communication).

${ }^{24}$ S. F. Boys and F. Bernardi, Mol. Phys. 19, 553 (1970).

${ }^{25}$ F. D. Murnaghan, Proc. Natl. Acad. Sci. U.S.A. 30, 244 (1944).

${ }^{26}$ H. M. Kandil, J. D. Greiner, and J. F. Smith, J. Am. Chem. Soc. 67, 341 (1982).

${ }^{27}$ M. Catti, R. Dovesi, A. Pavese, and V. R. Saunders, J. Phys. Condens. Matter 3, 4151 (1991); R. Dovesi, C. Roetti, C. Freyria-Pavia, M. Prencipe, and V. R. Saunders, Chem. Phys.
156, 11 (1991).

${ }^{28}$ A. Lichanot, M. Gelizé, C. Larrieu, and C. Pisani, J. Phys. Chem. Solids 52, 1155 (1991).

${ }^{29}$ D. M. Bishop, Group Theory and Chemistry (Clarendon, Oxford, 1973).

${ }^{30} \mathrm{Ph}$. Sautet (private communication).

${ }^{31}$ J. D. McCullough and K. N. Trueblood, Acta Crystallogr. 12, 507 (1959). 